NASA/TM-2001-210698

\title{
Rayleigh Scattering Diagnostic for Dynamic Measurement of Velocity and Temperature
}

R.G. Seasholtz

Glenn Research Center, Cleveland, Ohio

J. Panda

Modern Technologies Corporation, Middleburg Hts, Ohio 
Since its founding, NASA has been dedicated to the advancement of aeronautics and space science. The NASA Scientific and Technical Information (STI) Program Office plays a key part in helping NASA maintain this important role.

The NASA STI Program Office is operated by Langley Research Center, the Lead Center for NASA's scientific and technical information. The NASA STI Program Office provides access to the NASA STI Database, the largest collection of aeronautical and space science STI in the world. The Program Office is also NASA's institutional mechanism for disseminating the results of its research and development activities. These results are published by NASA in the NASA STI Report Series, which includes the following report types:

- $\quad$ TECHNICAL PUBLICATION. Reports of completed research or a major significant phase of research that present the results of NASA programs and include extensive data or theoretical analysis. Includes compilations of significant scientific and technical data and information deemed to be of continuing reference value. NASA's counterpart of peerreviewed formal professional papers but has less stringent limitations on manuscript length and extent of graphic presentations.

- TECHNICAL MEMORANDUM. Scientific and technical findings that are preliminary or of specialized interest, e.g., quick release reports, working papers, and bibliographies that contain minimal annotation. Does not contain extensive analysis.

- CONTRACTOR REPORT. Scientific and technical findings by NASA-sponsored contractors and grantees.
- CONFERENCE PUBLICATION. Collected papers from scientific and technical conferences, symposia, seminars, or other meetings sponsored or cosponsored by NASA.

- SPECIAL PUBLICATION. Scientific, technical, or historical information from NASA programs, projects, and missions, often concerned with subjects having substantial public interest.

- TECHNICAL TRANSLATION. Englishlanguage translations of foreign scientific and technical material pertinent to NASA's mission.

Specialized services that complement the STI Program Office's diverse offerings include creating custom thesauri, building customized data bases, organizing and publishing research results ... even providing videos.

For more information about the NASA STI Program Office, see the following:

- Access the NASA STI Program Home Page at http://www.sti.nasa.gov

- E-mail your question via the Internet to help@sti.nasa.gov

- Fax your question to the NASA Access Help Desk at 301-621-0134

- Telephone the NASA Access Help Desk at 301-621-0390

- Write to:

NASA Access Help Desk

NASA Center for AeroSpace Information 7121 Standard Drive

Hanover, MD 21076 
NASA/TM-2001-210698

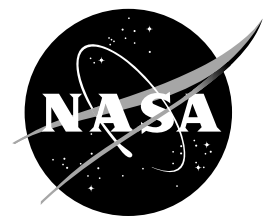

\section{Rayleigh Scattering Diagnostic for Dynamic Measurement of Velocity and Temperature}

R.G. Seasholtz

Glenn Research Center, Cleveland, Ohio

J. Panda

Modern Technologies Corporation, Middleburg Hts, Ohio

Prepared for the

37th Aerospace Sciences Meeting and Exhibit

sponsored by the American Institute of Aeronautics and Astronautics

Reno, Nevada, January 11-14, 1999

National Aeronautics and

Space Administration

Glenn Research Center 


\section{Acknowledgments}

We would like to acknowledge the diligent efforts of Mr. W. Trevor John, who was responsible for setting up and aligning the optical system used for this work.

Available from

NASA Center for Aerospace Information 7121 Standard Drive

Hanover, MD 21076

Price Code: A03
National Technical Information Service 5285 Port Royal Road Springfield, VA 22100 Price Code: A03 


\title{
Rayleigh Scattering Diagnostic for Dynamic Measurement of Velocity and Temperature
}

\author{
R.G. Seasholtz \\ National Aeronautics and Space Administration \\ Glenn Research Center \\ Cleveland, Ohio 44135 \\ J. Panda \\ Modern Technologies Corporation \\ Middleburg Hts., Ohio 44130
}

\begin{abstract}
$\underline{\text { Abstract }}$
A new technique for measuring dynamic gas velocity and temperature is described. The technique is based on molecular Rayleigh scattering of laser light, so no seeding of the flow is necessary. The Rayleigh scattered light is filtered with a fixed cavity, planar mirror Fabry-Perot interferometer. A minimum number of photodetectors were used in order to allow the high data acquisition rate needed for dynamic measurements. One photomultiplier tube (PMT) was used to measure the total Rayleigh scattering, which is proportional to the gas density. Two additional PMTs were used to detect light that passes through two apertures in a mask located in the interferometer fringe plane. An uncertainty analysis was used to select the optimum aperture parameters and to predict the measurement uncertainty due to photon shot-noise. Results of an experiment to measure the velocity of a subsonic free jet are presented.
\end{abstract}

Introduction

A variety of nonintrusive, laser based flow diagnostics (Rayleigh scattering, LDV, PIV, DGV, LIF) are routinely being applied for time average and instantaneous planar measurements of velocity, density, temperature, and species concentrations. However, an important class of measurements is not addressed by current laser diagnostics. High frequency response dynamic measurements of flow parameters cannot be made with existing techniques. This type of time history data is needed to determine, for example, density and velocity spectra, density-velocity correlations, and two-point correlations. Although Laser Doppler Velocimetry (LDV) can typically achieve data rates of a few tens of $\mathrm{kHz}$, it is difficult to achieve much higher rates without introducing larger amounts of seed material into the flow. In practice, LDV normally provides mean velocity and a measure of turbulence intensity. Furthermore, the measurements are random in time, which makes it difficult to obtain time history data needed for power spectra. In turbulent flows, LDV measurements are beset by a variety of so called biasing errors, caused by correlations between the measurement rate and flow properties. Planar techniques, such as PIV, provide a large number of simultaneous measurements in the measurement plane, but are generally limited to low sampling rates, determined by the pulse repetition rate of the laser and by the time needed to transfer image data from the camera.

New techniques are needed to provide nonintrusive, dynamic measurements that can provide data similar to that provided by hot wire anemometers. Since it unrealistic to expect (at least at the present time) to make measurements at a large number of locations and at a high sampling rate, it seems prudent to develop a laser diagnostic capable of point measurements at a high sampling rate. Such time resolved point measurements are in a form familiar to fluid dynamics researchers.

In this paper, we describe a laser diagnostic for dynamic measurement of gas properties based on molecular Rayleigh scattering. Because the measurement is based on molecular scattering, rather than particle scattering, no seed material need be injected into the flow under study. One difficulty with LDV and PIV is that they require the flow to be "seeded" with micron sized seed particles to provide a sufficient concentration of scattering centers. The reliance on seed particles presents a number of difficulties. It is difficult to inject a uniform cloud of seed into many test facilities. And the seed material must withstand the flow environment. For high temperatures, refractory seed materials are necessary. In addition, seed material can contaminate the facility, coating surfaces and windows. Facility engineers are often wary of the introduction of this foreign, often abrasive material, into their equipment. A further limitation of particle scattering methods is that the particles, although small, may not be able to follow large flow accelerations, thus introducing inaccuracy in the prediction of the gas velocity. 
These problems associated with particle scattering measurements are eliminated if molecular scattering is used, since the gas molecules that constitute the flow under study are used as the scattering centers. The simplest molecular scattering based diagnostic is Rayleigh scattering. The frequency spectrum of Rayleigh scattering is closely related to the velocity distribution of the scattering gas. The spectrum may be analyzed to determine temperature, density, and velocity. Density is simply proportional to the total scattered light; temperature is obtained from the width of the Rayleigh spectrum; and one component of velocity is proportional to the shift of the Rayleigh spectral peak from the frequency of the incident light. Because the spectral width is also a function of the molecular weight of the gases in the flow, knowledge of the gas composition is generally required. This, however, is not a concern in the proposed work, which is directed toward air flows where the composition is well defined. However, because velocity is determined from the peak of spectrum, it is independent of the gas composition. Rayleigh scattering is particularly suitable for measurement of supersonic and hypersonic velocities, where the mean molecular velocity (flow velocity) is greater than the random molecular velocity (temperature). One quantity of particular interest in the study of aerodynamically generated sound that can be obtained from Rayleigh scattering measurements is $\rho V$ (the product of gas density and velocity).

The key to obtaining high frequency response data is to combine a high power, continuous wave laser, or a high repetition rate pulsed laser, with high efficiency collection optics. Density measurements are relatively straightforward, since the Rayleigh scattered light is directly proportional to the density. Velocity and temperature measurements are more challenging because they must be determined from the spectral characteristics of the Rayleigh scattered light.

Both atomic/molecular vapor absorption filters and Fabry-Perot interferometers can be used to measure spectral characteristics that are related to the gas velocity and temperature. Iodine vapor is commonly used as an absorption medium because it has a rich absorption spectrum in the green wavelength region ${ }^{1}$. Use of absorption filters requires a tunable laser that can be tuned to a suitable absorption line. One approach for obtaining real-time velocity measurements is to use an absorption filter to analyze frequency modulated Rayleigh scattered light ${ }^{2,3}$. A Faraday cell has been used as a frequency discriminator for seeded flows ${ }^{4}$. Fabry-Perot interferometers, on the other hand, act as bandpass filters. They are quite flexible in that they can be used at any laser frequency, and the width of their passband can be easily adjusted. However, the transmission is also a function of the angle of the light beam in the Fabry-Perot interferometer cavity relative to the optical axis. Fabry-Perot etalons have been proposed for analyzing Rayleigh scattering from the atmosphere to obtain wind velocity and temperature ${ }^{5}$.

The approach taken in the present work is to use a Fabry-Perot interferometer with fixed mirror spacing. Characteristics of the transmitted light are measured using a minimum number of fast photo detectors. This allows a high sampling rate to achieve the desired dynamic response.

We first establish the lower bounds for uncertainty in the measurements of velocity and temperature caused by photon statistical noise. We then examine a particular scheme based on a Fabry-Perot interferometer and three photomultipler tubes and determine the lower bounds for measurement errors. A similar approach has previously been used to determine lower bounds for temperature and velocity uncertainties for measurements obtained with a scanning Fabry-Perot interferometer ${ }^{6}$, as well as for an imaging system ${ }^{7}$. The results of a preliminary experiment to evaluate this technique are then presented.

\section{Theory}

For the purposes of this paper we consider Rayleigh scattering from a low density gas, which has a Gaussian spectrum given by

$$
S\left(f-f_{o}\right) d f=\frac{2 \sqrt{\pi}}{a K} \exp \left\{-\left[\frac{2 \pi\left(f-f_{o}\right)-\mathbf{K} \cdot \mathbf{u}}{a K}\right]^{2}\right\} d f
$$

where $f_{\mathrm{o}}$ is the laser frequency and $\mathbf{u}$ is the mean gas velocity. Note that the spectral peak is shifted by a frequency proportional to the component of the bulk velocity in the $\mathbf{K}$ direction. The spectral width is proportional to the square root of the gas temperature. It is convenient to introduce the velocity component $u_{\mathrm{K}}$ $=\mathbf{K} \cdot \mathbf{u} / K$, which represents the measured velocity component. The interaction wave vector is $\mathbf{K}=\mathbf{k}_{\mathrm{s}}-\mathbf{k}_{\mathrm{o}}$ (with $\mathbf{k}_{\mathbf{0}}$ and $\mathbf{k}_{\mathbf{s}}$ being the wave vectors of the incident and scattered light), and $\mathrm{a}=(2 \kappa T / m)^{1 / 2}$ is the most probable molecular speed (with $\kappa$ being Boltzmann's constant, $\mathrm{m}$ the molecular mass, and $\mathrm{T}$ the gas temperature). (Note that $a=(2 / \gamma)^{1 / 2} c_{\mathrm{s}}$, where $c_{\mathrm{s}}$ is the speed of sound and $\gamma$ is the ratio of specific heats.).

The assumption of a Gaussian shaped Rayleigh scattering spectrum is only valid if

$$
y=\frac{p}{\eta K a} \ll 1
$$

where $p$ is the gas pressure and $\eta$ is the shear viscosity. For higher density gases $(y \sim 1)$, a more detailed kinetic 
theory model, such as the Tenti S6 model ${ }^{8}$ is required to describe the Rayleigh scattering spectrum. To simplify the analysis presented here, we use the Gaussian spectral model. However, as shown in Figure 1, the Tenti spectrum differs significantly from the Gaussian spectrum for the conditions encountered in this work ( $y$ $=0.79$ ). A more accurate analysis would use the Tenti spectral model.

Since Rayleigh scattering is a relatively weak process, the uncertainty in the measurements often is set by the photon statistical noise (shot noise), which determines the lower bound on measurement uncertainty. For example, the variance in the number of photoelectron counts for a Poisson process is equal to the mean number of counts. Thus the lower bound for the relative uncertainty in the measurement of gas density $\rho$, is equal to the square root of the variance divided by the mean number of counts. The relative uncertainties can be written ${ }^{6,9}$

$$
\frac{\sigma(\rho)}{\rho}=\left(\frac{1}{N_{R}}\right)^{1 / 2}, \quad \frac{\sigma(T)}{T}=\left(\frac{2}{N_{R}}\right)^{1 / 2}, \quad \sigma\left(u_{K}\right)=\frac{a}{\left(2 N_{R}\right)^{1 / 2}}
$$

These relations provide a lower bound for the measurement uncertainties, which can only be approached if the shot noise is the dominant noise and if an ideal instrument is used. Note that the lower bounds for the uncertainties of density, temperature, and velocity are all inversely proportional to the square root of the number of detected photons.

Estimates of the measurement uncertainty for the technique described here, where the Rayleigh scattered light is analyzed with a planar mirror FabryPerot interferometer, are obtained by numerically calculating the Cramer-Rao lower bound ${ }^{10}$. The variance of the estimate of a parameter $\alpha_{i}$ (e.g., temperature or velocity) is given by

$$
V\left(\alpha_{i}\right)=\left[\Gamma^{-1}\right]_{i i}
$$

where, for Poisson statistics, $\Gamma$ is the Fisher information matrix with elements

$$
\Gamma_{i j}=\sum_{q} \frac{1}{\left\langle N_{D_{q}}\right\rangle} \frac{\partial\left\langle N_{D_{q}}\right\rangle}{\partial \alpha_{i}} \frac{\partial\left\langle N_{D_{q}}\right\rangle}{\partial \alpha_{j}}
$$

and the summation is over the number of photodetectors. For example, in previous work ${ }^{11}$ using a CCD array detector, the summation would be over the pixels in the image being analyzed. For this work, however, a CCD camera cannot be used because of its slow readout rate (typically on the order of 1 second for scientific grade cameras). To achieve the high data acquisition rate desired for dynamic measurements, we use photomultipliers tubes (PMTs), which combine reasonable quantum efficiency with high gain and lownoise. One PMT is used to measure the total Rayleigh scattered light (i.e., the spectrally integrated light), which gives a measurement of the gas density. Two additional PMT's are used to detect the Rayleigh scattered light that is transmitted through a planar mirror interferometer.

Consider an experiment with the following parameters: a laser $(532 \mathrm{~nm})$ with output power $P_{o}=4$ $\mathrm{W}$, air at STP, probe volume length $L_{x}=1 \mathrm{~mm}, \mathrm{f} / 4$ collecting optics (i.e., solid collection angle $\Omega=0.05$ sr), and efficiency factor $\varepsilon=5 \%$. The rate of detected photons, given by

$$
\begin{aligned}
& N_{R}=\frac{\varepsilon P_{o} n L_{x} \lambda \Omega}{h c}\left(\frac{\mathrm{d} \sigma}{\mathrm{d} \Omega}\right) \sin ^{2} \chi \\
& =39 \text { million counts } / \mathrm{sec}
\end{aligned}
$$

In this equation, $n$ is the gas number density, $d \sigma / d \Omega$ is the differential scattering cross section, $\chi$ is the angle between the electric field vector of the (linearly polarized) incident light and the direction of the scattered light, $h$ is Planck's constant, and $c$ is the velocity of light. If we wish to sample at a $10 \mathrm{kHz}$ rate, the total number of detectable photons in each sampling period would be 3900. Equation 2 gives the lower bound for measurement of density, temperature and velocity for each sample period:

$\frac{\sigma(\rho)}{\rho}=1.6 \%, \quad \frac{\sigma(T)}{T}=2.3 \%, \quad \sigma\left(u_{K}\right)=3.6 \mathrm{~m} / \mathrm{sec}$

This shows that high sampling rate Rayleigh scattering measurements are at least feasible. It must be emphasized, however, that these values represent the best possible measurements given an ideal instrument. In practice, we are limited to instruments, such as the Fabry-Perot interferometer used here, which result in significantly higher uncertainties, as described below. Achievement of the uncertainties given above will require a photoelectron detection rate considerably above those given in this example. This can be achieved by using higher laser power, larger collection f-number, or higher quantum efficiency photodetectors.

\section{Optical configuration}

In order to perform an uncertainty analysis, we first describe the setup (fig. 2) used for the experimental work. Light from a $5 \mathrm{~W}, 532 \mathrm{~nm}$, single-frequency, 
Nd:Vanadate CW laser is focused by lens L1 $(300 \mathrm{~mm}$ focal length) to a $150 \mu \mathrm{m}$ diameter beam at the probe volume. The $90^{\circ}$ Rayleigh scattered light is collected and collimated by lens L2 (250 mm focal length) and is then split into two paths with an uncoated optical flat. About $10 \%$ of the light is reflected and focused by lens L3 onto PMT 1 (type R3896). This detected signal is proportional to gas density. Light in the second path is directed through a planar mirror Fabry-Perot interferometer $(70 \mathrm{~mm}$ dia. mirrors, $90 \%$ reflectivity, $15 \mathrm{GHz}$ free spectral range). The light exiting the interferometer is focused by the fringe forming lens, L4 (135 mm focal length, $\mathrm{f} / 2$ ). An addition lens L5 (50 $\mathrm{mm}$ focal length, $\mathrm{f} / 1.2$ ) is used to form a magnified image of the fringe pattern. The effective focal length of combination of lenses L4 and L5 is $1823 \mathrm{~mm}$. The fringe pattern formed at the focal plane of this lens pair is analyzed to determine the velocity and temperature. Typical calculated images of the inner fringe of Rayleigh scattered light from a gas with zero bulk velocity and a temperature of $300 \mathrm{~K}$ are shown in figure 3. One image shows Rayleigh scattered light from an extended region, which results in the full circular fringe; the other image shows the image resulting from a line source as was used in the experiment.

Because, in the experiment, the laser beam is focused to a line in the probe volume, the fringe exists only along that line. Also, to simplify the setup and to use a minimum number of photodetectors, only one half of the fringe pattern is utilized. A mask having two apertures, shown as white rectangles in figure 3 and referred to as the "inner" and "outer" regions, is located in the fringe plane. Light passing through the inner and outer regions is directed to two PMT's (type 8645). As the velocity and temperature of the gas change, the relative distribution of the light detected by the two PMT's changes. Note that a flow in the direction opposite the $\mathbf{K}$ vector results in a negative frequency shift. Use of additional detectors would improve the measurement uncertainty, but at an increase in the system complexity.

Additional optics were included to provide for a reference image consisting of light at the unshifted laser frequency. To accomplish this, several components could be placed in the optical path. When placed in the beam, mirror $\mathrm{M}$ directed laser light through neutral density filter NDF onto a diffuser assembly that could be inserted at the measurement location. This assembly consisted of a diffuser that scattered light into the collection optics and an iris to limit the spatial extent of the light. Finally, a prism assembly could be placed in the light path between the Fabry-Perot interferometer and the PMT's. This served to direct light into a standard video camera. The video signal from this camera was digitized by a frame grabber card in a 486 PC. A computer program analyzed this image and generated signals to control the Fabry-Perot mirror alignment. The mirror $\mathrm{M}$, diffuser assembly, and prism assembly were mounted on remotely controlled pneumatic linear actuators.

\section{Uncertainty analysis}

We now calculate the Cramer-Rao lower bounds for velocity and temperature measurements based on the experimental setup described above. This allows us to conduct parametric studies to determine the optimum configuration for Fabry-Perot interferometer and for the light detection system. The observed fringe pattern for Rayleigh scattered light can be expressed as

$$
<N_{D q}>=\iint A_{R} S_{R}(f) I_{F P}(f, \theta) d f d \Omega
$$

where $\left\langle N_{D q}\right\rangle$ is the expected number of detected photons in the $q^{\text {th }}$ area, $A_{R}$ is the number of photons that would be detected if the Fabry-Perot interferometer were removed from the system, and $I_{F P}$ is the FabryPerot instrument function given by ${ }^{12}$

$$
I_{F P}(\psi)=\left[1+F \sin ^{2}\left(\frac{\psi}{2}\right)\right]^{-1}
$$

where $\psi$ is the phase change (neglecting any phase change on reflection) of the light between successive reflections given by

$$
\psi\left(f, \theta_{r}\right)=\frac{4 \pi f \mu d \cos \theta_{r}}{c}
$$

Here, $\mu$ is the refractive index of the medium in the Fabry-Perot cavity, $d$ is the Fabry-Perot mirror spacing, $\theta_{r}$ is the angle between the ray and the optic axis, and $F$ $=1 /\left(\sin ^{2}\left(\pi / 2 N_{E}\right)\right.$ where $N_{E}$ is the effective finesse. In general, the image of a monochromatic extended source located in the object plane consists of a series of unequally spaced concentric rings. In this work, however, the field of view is restricted to include only the inner fringe as shown in figure 3.

We now calculate the lower bounds for velocity and temperature measurement uncertainties based on the detected light from the inner and outer regions. The uncertainties are evaluated as a function of two parameters $n_{o}$ and $n_{f}$, which characterize the two active regions in the fringe plane. Because the number of photoelectron counts from the two regions is a function of both temperature and velocity, a 
measurement of the photoelectron counts from a single region cannot be used to determine velocity.

For a given wavelength $\lambda_{o}$, the order of interference at the center of the fringe pattern is given by $2 \mu d=\left(n+n_{c}\right) / \lambda_{o}$, where $n$ and $n_{c}$ represent the integral and fractional parts of the order, respectively ${ }^{12}$. A bright fringe is located on the optical axis if $n_{c}=0$. Off axis, the order of interference at angle $\theta$ is given by $2 \mu d \cos \theta=\left(n+n_{c}-\right.$ $\left.n_{k}\right) / \lambda_{\mathrm{o}}$, where $n_{k}$ represents the change in the order of interference between optical axis and the angular radius $\theta$. (Note that a bright fringe occurs at $n_{k}=n_{c}$ ). For small angles, $n_{\mathrm{k}}=\mu d \theta^{2} / \lambda_{\mathrm{o}}$. The spectral interval included in the region from the optical axis out to $\theta$ is $n_{k} \cdot F S R$, where the free spectral range $F S R=c / 2 d$. It follows that the spectral interval between orders $n_{k l}$ and $n_{k 2}$ is $\left(n_{k 2}-n_{k 1}\right) F S R$. For the purpose of analysis, it is convenient to use the fractional order $n_{k}$ to describe locations in the Fabry-Perot fringe plane. Because we are working in air, we assume that $\mu=1$ in the remainder of this analysis.

We can now define the boundaries of the fringe plane mask, as shown in figure 3 , in terms of fractional orders: $n_{o}$ is the fractional order of the fringe corresponding to the laser wavelength; $\left(n_{o}-n_{f}\right)$ and $\left(n_{o}\right.$ $+n_{f}$ ) correspond to the inner boundary and outer boundaries of the central stop, respectively; and $n_{m}$ corresponds to the outer boundary of the aperture. (Here we assume that the central stop of the mask is located at fringe position.) Note that the actual radius of a fractional order $n_{x}$ in the focal plane of the fringe forming lens is given by the product the angle $\theta_{x}=\left(\lambda_{\mathrm{o}}\right.$ $\left.\mathrm{n}_{\mathrm{x}} / d\right)^{1 / 2}$ and the focal length of fringe forming lens. Also note that the image of the fringe pattern projected back to the probe volume is similarly given by the product of the angle and focal length of the collimating lens. For the mask used in this work, the active area of the probe volume is about $1 \mathrm{~mm}$ long.

The lower bounds for velocity and temperature were numerically evaluated as a function of the parameters $n_{\mathrm{o}}$ and $n_{\mathrm{f}}$, which characterize the dimensions of the two active areas. The results are shown in figure 4. These plots are for a particular velocity $(0 \mathrm{~m} / \mathrm{sec})$ and temperature $(300 \mathrm{~K})$. The order of the outer boundary of the outer aperture was $n_{m}=0.341$. Note that the minimum uncertainties for velocity and temperature occur at different values of $n_{\mathrm{o}}$ and $n_{\mathrm{f}}$. It can be seen that the values of $n_{\mathrm{o}}$ and $n_{\mathrm{f}}$ that result in a minimum velocity uncertainty (about $13 \mathrm{~m} / \mathrm{sec}$ ) are 0.087 and 0.027 , respectively. On the other hand, the minimum temperature uncertainty (about $34 \mathrm{~K}$ ) corresponds to $n_{\mathrm{o}}$ and $n_{\mathrm{f}}$ values of 0.14 and 0.08 , respectively. Other temperatures and velocities would result in other optimum values of $n_{\mathrm{o}}$ and $n_{\mathrm{f}}$. For this study, we chose the configuration that results in the minimum velocity uncertainty at the expense of higher temperature uncertainty. We show in figure 5 the velocity and temperature uncertainties as a function of velocity and temperature for $n_{\mathrm{o}}=0.087$ and $n_{\mathrm{f}}=0.039$ (close to values that result in minimum velocity uncertainty at $0 \mathrm{~m} / \mathrm{sec}$ and $300 \mathrm{~K}$ ). The minimum velocity uncertainty is about a factor of four greater than that calculated above for an ideal instrument, and the minimum temperature uncertainty is about a factor of five greater than that of an ideal instrument. (Recall, however, that the minimum velocity and temperature uncertainties do not occur for the same values of $n_{\mathrm{o}}$ and $n_{\mathrm{f} .) \text {. }}$

\section{Experiment}

The optical system described above and shown in figure 2 (with the laser output power equal $5 \mathrm{~W}$ ) was used to measure the flow in the potential core of a subsonic free jet (15.9 $\mathrm{mm}$ exit diameter). The laboratory compressed air supply used for the jet was passed through a $0.2 \mu \mathrm{m}$ filter to remove particles. As shown in figure 2 , the jet axis bisected the incident and scattered light directions. With this configuration, the measured velocity component was the jet axial component. Since the flow velocity was opposite the $\mathbf{K}$ vector, the frequency shift was negative. Thus the Fabry-Perot interferometer fringe diameters of the Rayleigh scattered light decreased as the flow velocity increased. That is, as the velocity increased, the signal from the inner region increased, while the signal from the outer region decreased. An electronic pressure gauge was used to measure the total pressure in the nozzle plenum. These pressure measurements were used to calculate the flow velocity and temperature using the isentropic flow relations.

Signals from the three PMT's were digitized at a $50 \mathrm{kHz}$ rate with a digital oscilloscope. For this work, the PMT signals were fed directly into the $1 \mathrm{M} \Omega$ oscilloscope inputs. The data record length was $5 \mathrm{sec}$, which corresponded to 250,000 samples on each of the three channels. These data were stored on the oscilloscope's hard disk for later processing.

\section{Data processing}

The data processing scheme was based on the model described above. To account for gas density changes, the signals from PMT2 and PMT3 were first normalized by the signal from PMT1. The next step was to find the gain factor that related the measured signal voltages to the number of photoelectrons counts obtained from the model. To do this, an initial data set was taken at a known flow condition, usually zero, or close to zero, velocity and a known temperature. The 
gain factors were obtained by matching the photoelectron counts for the two channels from the model with the measured values. Note that this calibration only requires a measurement at a single flow condition. The data taken at other flow conditions were then analyzed using the theoretical model to obtain both velocity and temperature. Figure 6 shows the grid of predicted velocity and temperature as a function of the signals from inner and outer regions. The measured values of the inner and outer signals (example shown as squares on figure 6) were converted to velocity and temperature by interpolating between the constant temperature and the constant velocity contours. Note that the measured values generally follow the model. As discussed above, the system was optimized for velocity measurement near zero velocity.

\section{$\underline{\text { Experimental results }}$}

A typical time record of the digitized signals from the three PMT's is shown in figure 7. The bandwidth of the signals was set by the RC time constant of the PMT's and associated coaxial cables' capacitance and the $1 \mathrm{M} \Omega$ oscilloscope input impedance (1.12 ms for PMT 2 and PMT 3, determined using a pulsed LED as the light source). In figure 8, we show an example of a time record obtained while the jet total pressure was varied. The inner and outer signals are shown along with the velocity obtained from them. This illustrates the real-time capability of the technique. In figure 9, we show two sets of velocity data taken at different fixed jet velocities. The velocity obtained from the Rayleigh scattering measurements is shown along with the velocity obtained from the measured total pressure and isentropic flow relations. The flow velocity range was from zero to about $-100 \mathrm{~m} / \mathrm{sec}$ (negative because the frequency shift is negative). The point used to obtain the calibration is shown on each set. It should be noted that the total pressure measurement only had a resolution of 0.01 psia, thus the isentropic velocities could have a larger error at low velocities. These results are based on the average time record over the 5 second time interval. The first set shows good agreement, including the last point, which was taken at the same flow condition as the first point. (The first point was used as the calibration point.) The second set of measurements shows poor agreement if the first point is used as the calibration point. If the last point, however, is used as the calibration point, better agreement is obtained. The disagreement at the low velocity may be a result of the inaccuracies in the velocity obtained from the total pressure at low velocity.

\section{Concluding remarks}

Molecular Rayleigh scattering appears to be a feasible technique for the dynamic measurement of gas density, velocity, and temperature. There are, however, several requirements for obtaining successful measurements. First, a high power laser source is needed to generate a sufficiently large Rayleigh scattering signal to overcome the inherent statistical photon noise. Second, the maximum amount of the Rayleigh scattered light must be collected and detected, which means fast collection optics coupled with large diameter Fabry-Perot mirrors should be used with lownoise, high quantum efficiency photodetectors. The Fabry-Perot interferometer is extremely sensitive to any outside disturbances, including acoustic noise, mechanical vibration, and temperature changes. It is highly desirable to locate the Fabry-Perot in a quiet, stable environment, with the Rayleigh scattered light transmitted through fiber optics from the test facility. An automatic system for maintaining the interferometer alignment is also essential.

Two requirements must be met for the successful implementation of the technique described. One is the elimination of particles in the flow. The other is preventing any stray scattered laser light from reaching the photodetectors. These requirements will probably restrict this technique to unconfined flows unless extraordinary measures are taken. It should be noted that particle and stray light can be handled if more details of the spectrum are measured, which means the use of additional detectors. The system described here was designed to use the minimum number of detectors.

\section{$\underline{\text { References }}$}

${ }^{1}$ Elliott, G.S., and Samimy, M., "Rayleigh scattering technique for simultaneous measurements of velocity and thermodynamic properties", AIAA J., Vol. 34, 1996, pp. 2346-2352.

${ }^{2}$ Grinstead, J.H., Finkelstein, N.D., Lempert, W.R., and Miles, R.B., "Frequency-modulated filtered Rayleigh scattering (FM-FRS): a new technique for real-time velocimetry", AIAA $34^{\text {th }}$ Aerospace Sciences Meeting, AIAA-96-0302, Reno, NV, Jan15-18, 1996.

${ }^{3}$ Mach, J., and Varghese, P.L., "Velocity measurements using filtered Rayleigh scattering of near-IR diode lasers", AIAA $36^{\text {th }}$ Aerospace Sciences Meeting, AIAA-98-0510, Reno, NV, Jan12-15, 1998. 
${ }^{4}$ Crafton, J., Messersmith, N., and Sullivan, J.P., "Filtered Doppler velocimeter: development of a point system", AIAA $36^{\text {th }}$ Aerospace Sciences Meeting, AIAA-98-0509, Reno, NV, Jan12-15, 1998.

${ }^{5}$ Korb, C.L., Gentry, B.M., and Weng, C.Y., "Edge technique: therory and application to the lidar measurement of atmospheric wind", Appl. Opt., Vol. 31, 1992, pp. 4202-4213.

${ }^{6}$ Seasholtz, R.G., High-speed anemometry based on spectrally resolved Rayleigh scattering, Fourth International Conference on Laser Anemometry, Cleveland, Ohio, 1991 [also NASA TM-104522].

${ }^{7}$ Seasholtz, R.G., "2D velocity and temperature measurements in high speed flows based on spectrally resolved Rayleigh scattering”, New Trends in Instrumentation for Hypersonic Research, A. Boutier, Ed., pp. 399-408, Kluwer Academic Publishers, Boston, 1993.

${ }^{8}$ Tenti, G., Boley, C.D. \& Desai, R.C., On the kinetic model description of Rayleigh Brillouin scattering from molecular gases, Can. J. Phys. 52, 1974, pp. 285-290.

${ }^{9}$ Seasholtz R.G. \& Lock, J.A., Gas temperature and density measurements based on spectrally resolved Rayleigh-Brillouin scattering, NASA Langley

Measurement Technology Conference, Hampton, VA, 1992.

${ }^{10}$ Whalen, A.D., Detection of Signals in Noise, Academic Press, New York, 1971, pp. 324-231.

${ }^{11}$ Seasholtz, R.G., Rayleigh scattering diagnostic for measurement of temperature and velocity in harsh environments, AIAA $36^{\text {th }}$ Aerospace Sciences Meeting, AIAA-98-0206, Reno, NV, Jan12-15, 1998.

${ }^{12}$ Vaughan, J.M., The Fabry Perot Interferometer, History, Theory, Practice and Applications, Adam Hilger, Bristol, 1989, pp. 89-112. 


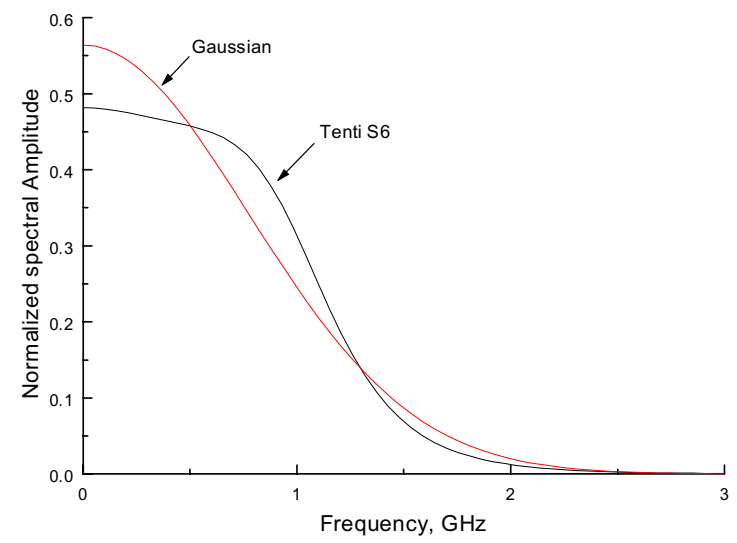

Fig. 1 - Comparison of Gaussian and Tenti S6 Rayleigh scattering spectra; y=0.79.

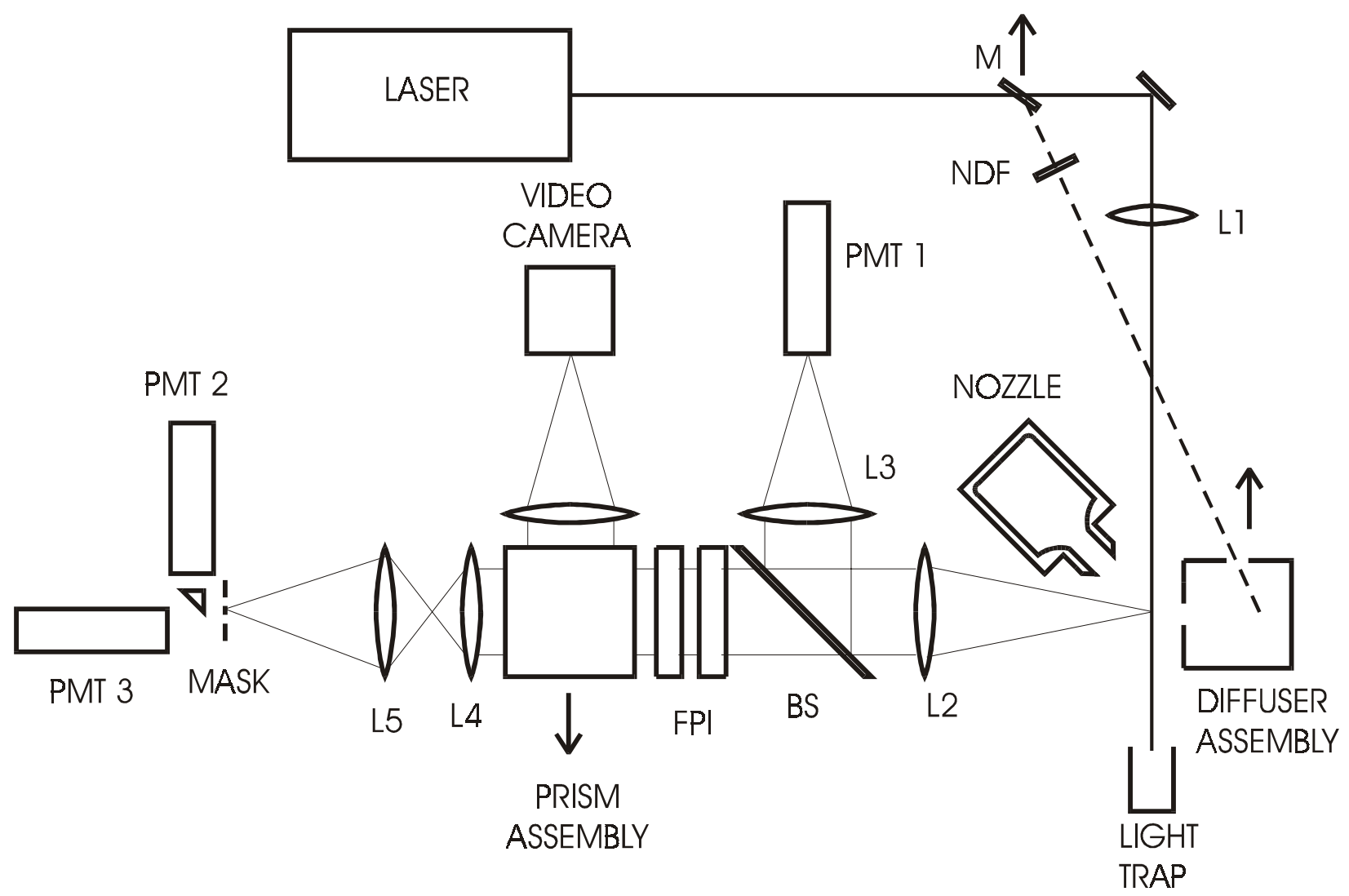

Fig. 2 - Setup for Dynamic Rayleigh scattering experiment. 

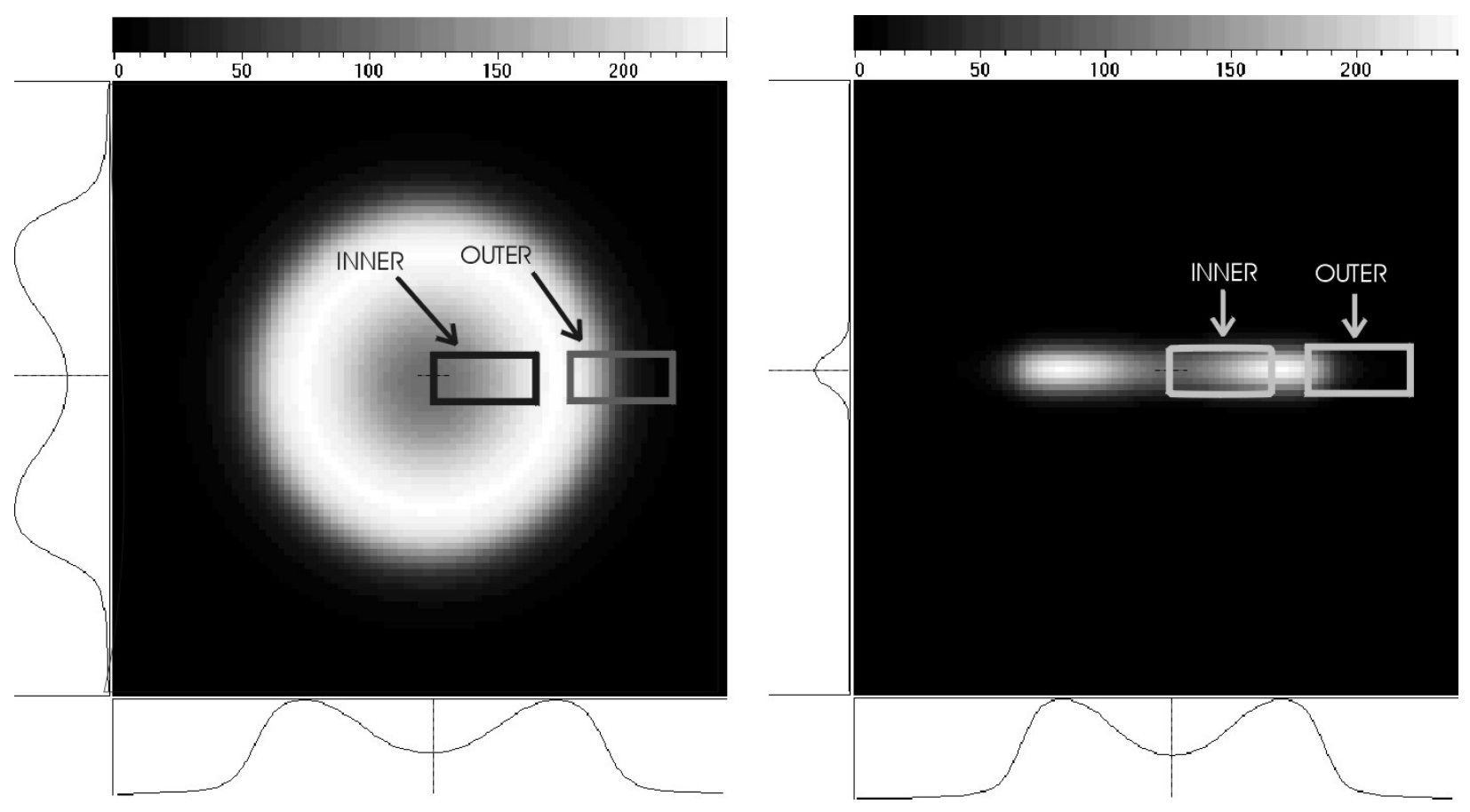

Fig. 3 - Fabry-Perot interferometer fringe pattern with mask showing two active areas; $n_{o}=0.087, n_{f}=0.039, n_{m}=$ 0.341; left image shows full fringe for extended source; right image shows fringe for line source used in experiment. 


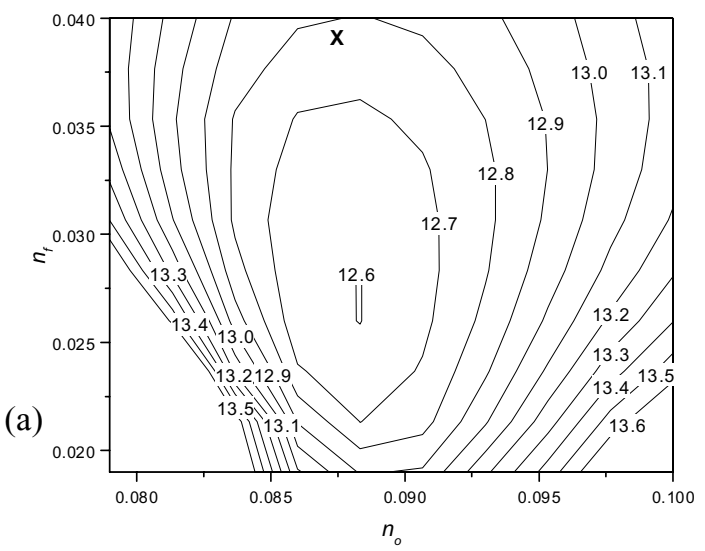

(b)
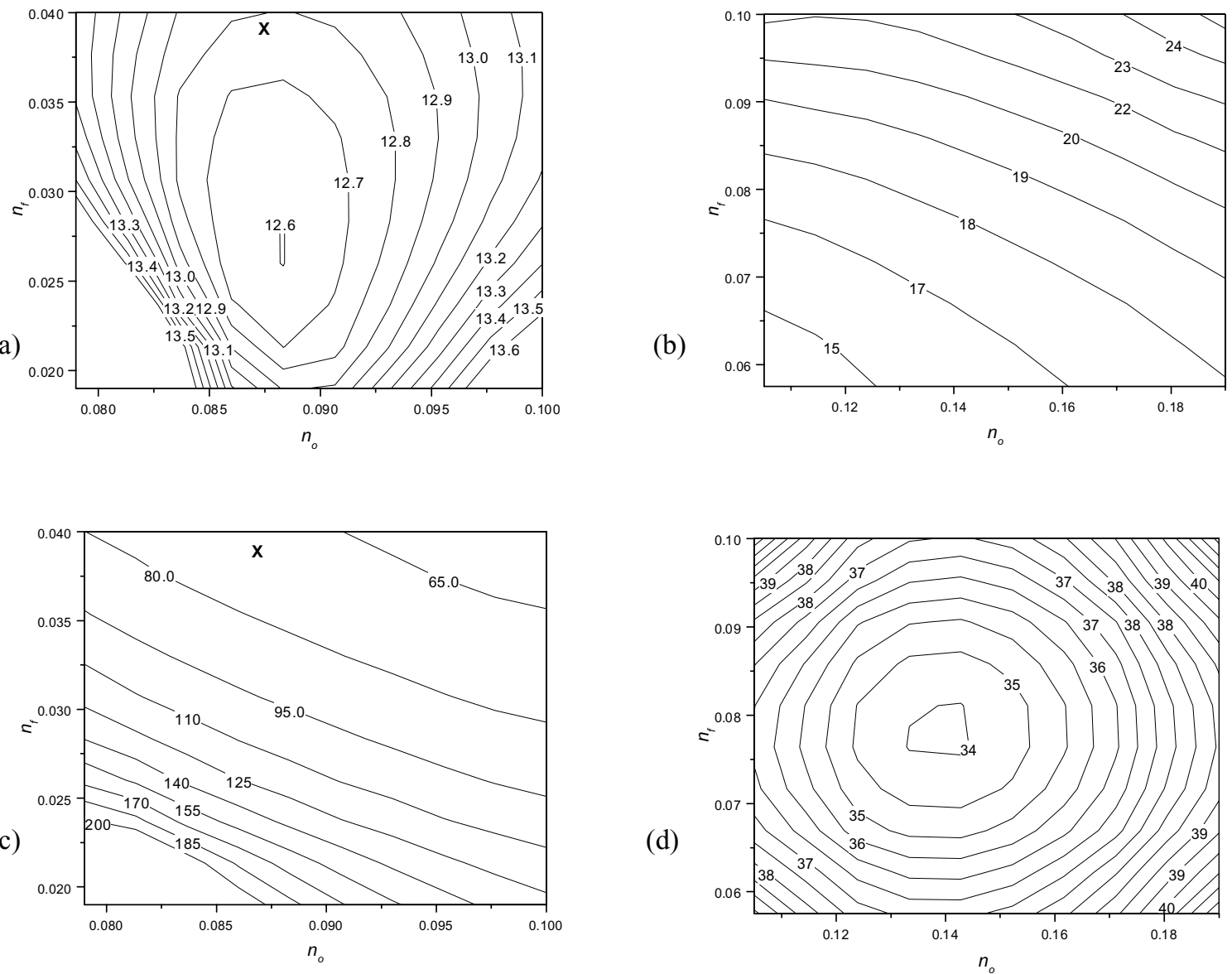

Fig. 4 - Lower bounds for uncertainties for velocity and temperature as a function of $n_{o}$ and $n_{f}$. (a) and (b) show contours of velocity uncertainty in $\mathrm{m} / \mathrm{sec}$; (c) and (d) show contours of temperature uncertainty in $\mathrm{K} ; \mathbf{X}$ on (a) and (c) at values of $n_{o}$ and $n_{f}$. used for experiment

(a)

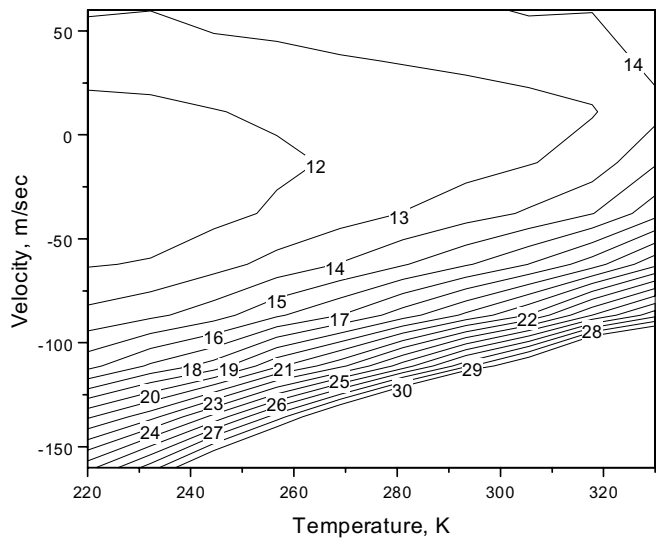

(b)

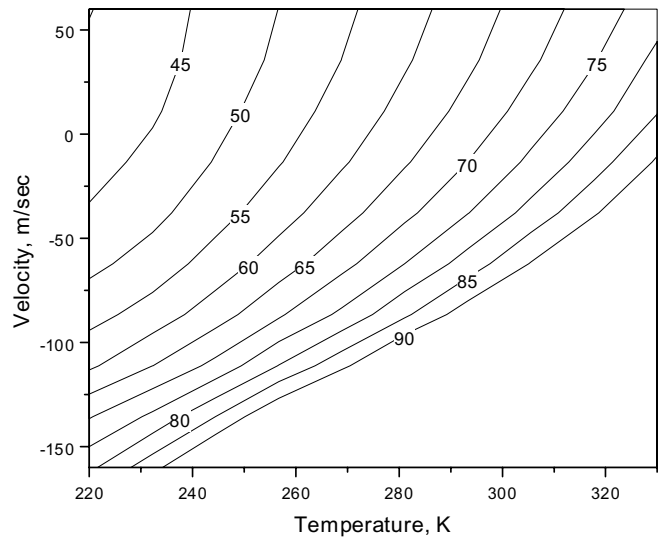

Fig. 5 - Uncertainty for velocity (a) and temperature (b) as a function of velocity and temperature for $n_{o}=0.087$ and $n_{f}=0.039$. 


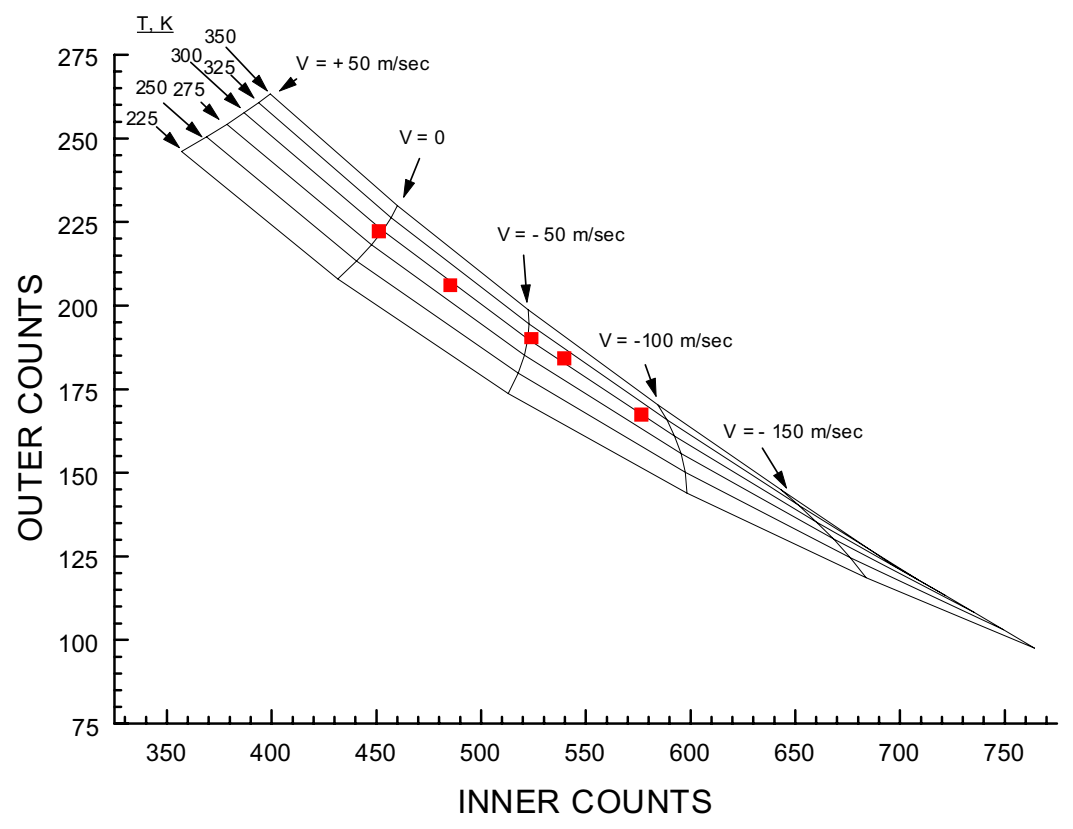

Fig. 6 - Plot of signal from inner and outer active regions as a function of velocity and temperature for $n_{o}=0.087$ and $n_{f}=0.039$. Squares mark location of experimental measurements.

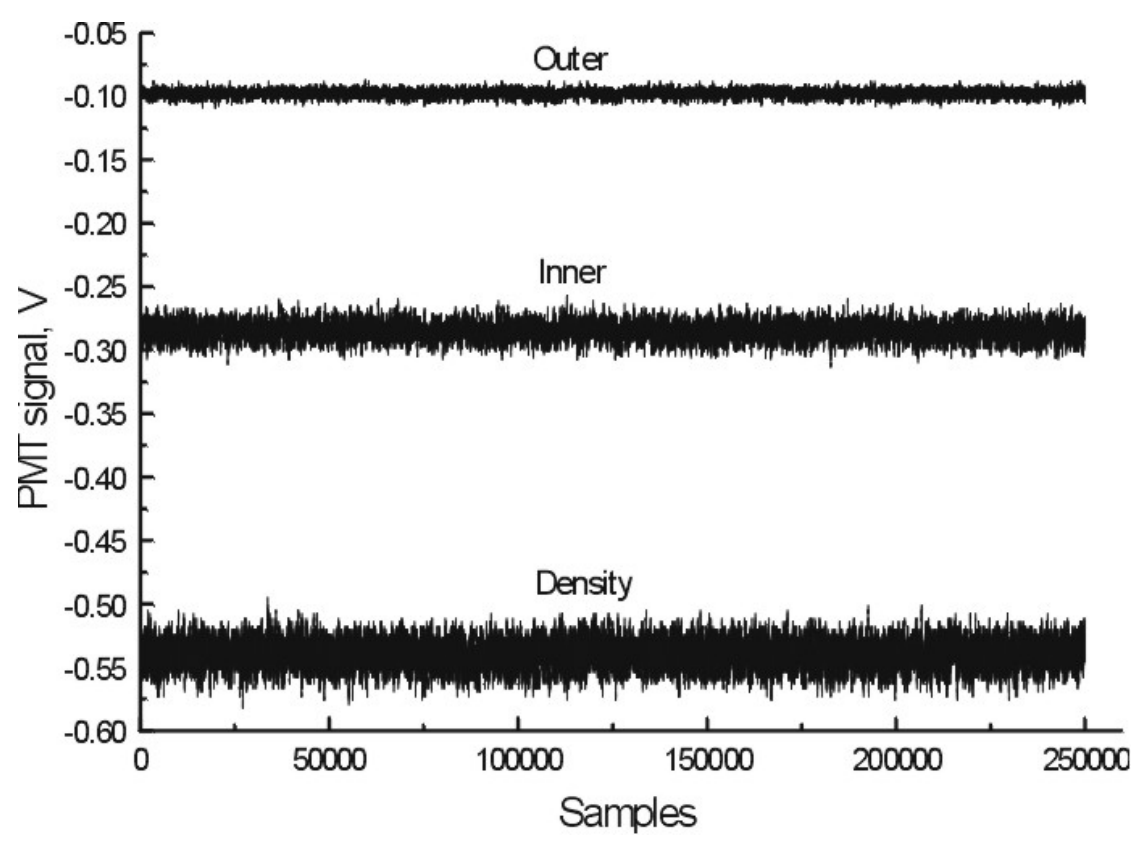

Fig. 7 - Typical PMT signals (density is divided by 10), sampling rate $=50 \mathrm{kHz}$. 

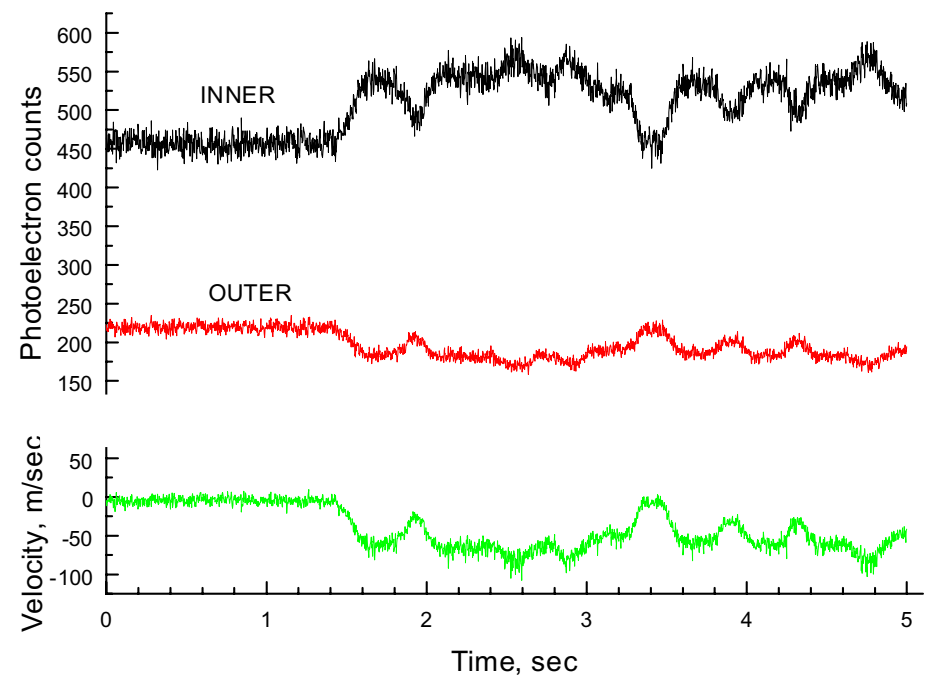

Fig. 8 - Time history of signal from two PMT's and velocity obtained from these signals. Velocity was manually changed over the time period.
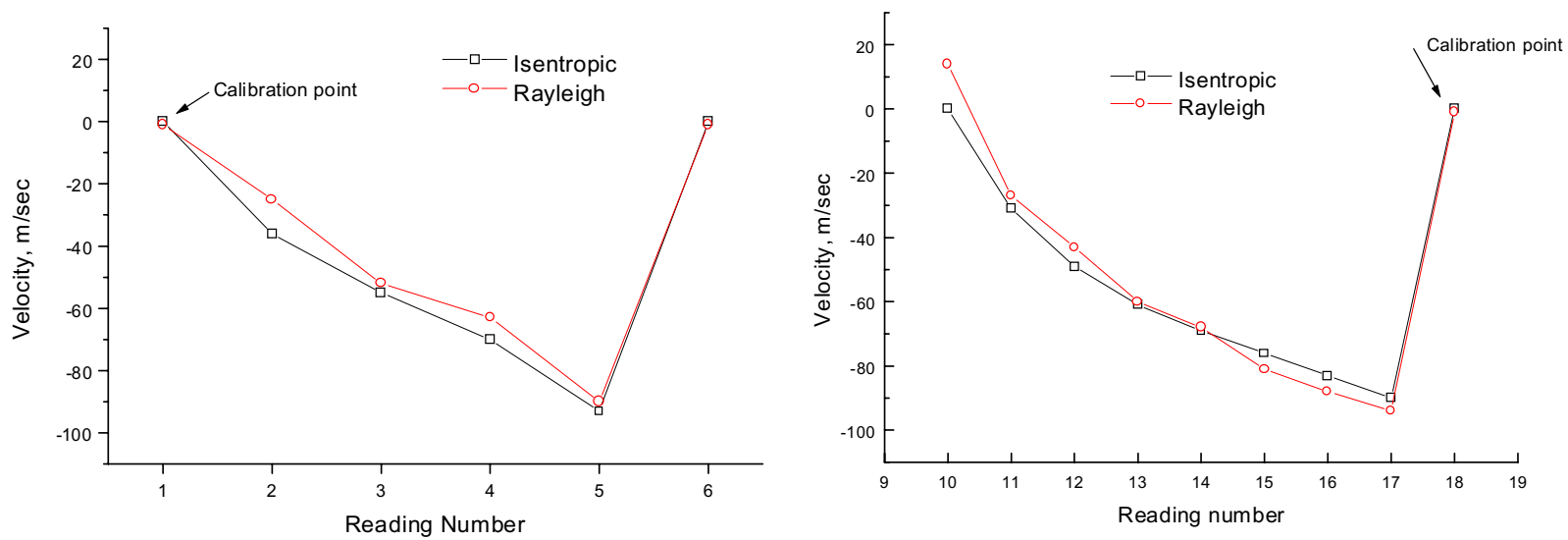

Fig. 9 - Comparison between time average velocity obtained from isentropic flow relations and from Rayleigh scattering measurements. 
Public reporting burden for this collection of information is estimated to average 1 hour per response, including the time for reviewing instructions, searching existing data sources, gathering and maintaining the data needed, and completing and reviewing the collection of information. Send comments regarding this burden estimate or any other aspect of this collection of information, including suggestions for reducing this burden, to Washington Headquarters Services, Directorate for Information Operations and Reports, 1215 Jefferson Davis Highway, Suite 1204, Arlington, VA 22202-4302, and to the Office of Management and Budget, Paperwork Reduction Project (0704-0188), Washington, DC 20503.

\begin{tabular}{|l|l|l}
\hline 1. AGENCY USE ONLY (Leave blank) & $\begin{array}{c}\text { 2. REPORT DATE } \\
\text { March } 2001\end{array}$ & $\begin{array}{c}\text { 3. REPORT TYPE AND DATES COVERED } \\
\text { Technical Memorandum }\end{array}$ \\
\hline
\end{tabular}

4. TITLE AND SUBTITLE 5. FUNDING NUMBERS

Rayleigh Scattering Diagnostic for Dynamic Measurement of Velocity and Temperature

6. AUTHOR(S)

WU-274-00-00-00

R.G. Seasholtz and J. Panda

7. PERFORMING ORGANIZATION NAME(S) AND ADDRESS(ES)

National Aeronautics and Space Administration

John H. Glenn Research Center at Lewis Field

Cleveland, Ohio 44135-3191

8. PERFORMING ORGANIZATION REPORT NUMBER

E-12629

9. SPONSORING/MONITORING AGENCY NAME(S) AND ADDRESS(ES)

National Aeronautics and Space Administration

Washington, DC 20546-0001

10. SPONSORING/MONITORING AGENCY REPORT NUMBER

NASA TM-2001-210698

AIAA-99-0641

\section{SUPPLEMENTARY NOTES}

Prepared for the 37th Aerospace Sciences Meeting and Exhibit sponsored by the American Institute of Aeronautics and Astronautics, Reno, Nevada, January 11-14, 1999. R.G. Seasholtz, NASA Glenn Research Center; J. Panda, Modern Technologies Corporation, Middleburg Hts, Ohio 44130. Responsible person, Richard G. Seasholtz, organization code 5520, 216-433-3754.

12a. DISTRIBUTION/AVAILABILITY STATEMENT 12b. DISTRIBUTION CODE

Unclassified - Unlimited

Subject Category: 35

Distribution: Nonstandard

Available electronically at http://gltrs.grc.nasa.gov/GLTRS

This publication is available from the NASA Center for AeroSpace Information, 301-621-0390.

13. ABSTRACT (Maximum 200 words)

A new technique for measuring dynamic gas velocity and temperature is described. The technique is based on molecular Rayleigh scattering of laser light, so no seeding of the flow is necessary. The Rayleigh scattered light is filtered with a fixed cavity, planar mirror Fabry-Perot interferometer. A minimum number of photodetectors were used in order to allow the high data acquisition rate needed for dynamic measurements. One photomultiplier tube (PMT) was used to measure the total Rayleigh scattering, which is proportional to the gas density. Two additional PMTs were used to detect light that passes through two apertures in a mask located in the interferometer fringe plane. An uncertainty analysis was used to select the optimum aperture parameters and to predict the measurement uncertainty due to photon shot-noise. Results of an experiment to measure the velocity of a subsonic free jet are presented.

\section{SUBJECT TERMS}

Rayleigh scattering; Fabry-Perot interferometers

16. PRICE CODE

$\mathrm{A} 03$

\begin{tabular}{|c|c|}
\hline $\begin{array}{c}\text { 17. SECURITY CLASSIFICATION } \\
\text { OF REPORT } \\
\text { Unclassified }\end{array}$ & $\begin{array}{c}\text { 18. SECURITY CLASSIFICATION } \\
\text { OF THIS PAGE } \\
\text { Unclassified }\end{array}$ \\
\hline
\end{tabular}

NSN 7540-01-280-5500

19. SECURITY CLASSIFICATION
OF ABSTRACT
Unclassified

Standard Form 298 (Rev. 2-89)

Prescribed by ANSI Std. Z39-18 298-102 